\title{
Rational Secret Sharing, Revisited
}

\author{
S. Dov Gordon* Jonathan Katz ${ }^{* \dagger}$
}

July 11, 2006

\begin{abstract}
We consider the problem of secret sharing among $n$ rational players. This problem was introduced by Halpern and Teague (STOC 2004), who claim that a solution is impossible for $n=2$ but show a solution for the case $n \geq 3$. Contrary to their claim, we show a protocol for rational secret sharing among $n=2$ players; our protocol extends to the case $n \geq 3$, where it is simpler than the Halpern-Teague solution and also offers a number of other advantages. We also show how to avoid the continual involvement of the dealer, in either our own protocol or that of Halpern and Teague.

Our techniques extend to the case of rational players trying to securely compute an arbitrary function, under certain assumptions on the utilities of the players.
\end{abstract}

\section{Introduction}

The classical problem of $t$-out-of-n secret sharing $[13,2]$ involves a "dealer" $D$ who wishes to entrust a secret $s$ to a group of $n$ players $P_{1}, \ldots, P_{n}$ so that (1) any group of $t$ or more players can reconstruct the secret without further intervention of the dealer, yet (2) any group of fewer than $t$ players has no information about the secret. As an example, consider the scheme due to Shamir [13]: assume the secret $s$ lies in a finite field $\mathbb{F}$, with $|\mathbb{F}|>n$. The dealer chooses a random polynomial $f(x)$ of degree at most $t-1$ subject to the constraint $f(0)=s$, and gives the "share" $f(i)$ to player $P_{i}$ (for $\left.i=1, \ldots, n\right)$. Any set of $t$ players can recover $f(x)$ (and hence $s$ ) by broadcasting their shares and interpolating the polynomial; furthermore, no set of fewer than $t$ players can deduce any information about $s$.

The implicit assumption above is that at least $t$ players are willing to cooperate and pool their shares ${ }^{1}$ when it is time to recover the secret; equivalently, at least $t$ players are honest but up to $n-t$ players may be arbitrarily malicious. Halpern and Teague [7] consider a scenario in which players are neither completely honest nor arbitrarily malicious, but instead all players are assumed to be rational (however, up to $n-t$ players may be unavailable at the time the secret is to be recovered). Depending on the utility functions of the players, Shamir's protocol may no longer succeed in this scenario [7]. Specifically, assume that all players prefer to learn the secret above all else, but otherwise prefer that the fewest number of other players learn the secret. (We will treat the utilities of the players more precisely later in the paper.) Given these utility functions, no

\footnotetext{
${ }^{*}$ Department of Computer Science, University of Maryland. \{gordon, jkatz\}@cs.umd.edu

${ }^{\dagger}$ Research supported by NSF Trusted Computing grants \#0310499 and \#0310751; NSF CAREER award \#0447075; and US-Israel Binational Science Foundation grant \#2004240.

${ }^{1}$ We assume adversarial behavior is limited to refusal to cooperate, and ignore the case that a player reports an incorrect share. In the present context, reporting an incorrect share is easily prevented by having the dealer sign the shares.
} 
player has any incentive to reveal their share. Consider $P_{1}$ : if strictly fewer than $t-1$ other players reveal their shares to the rest of the group, then no one learns the secret regardless of whether $P_{1}$ reveals his share or not. If more than $t-1$ players reveal their shares, then everyone learns the secret and $P_{1}$ 's actions again have no effect. On the other hand, if exactly $t-1$ other players reveal their shares, then $P_{1}$ learns the secret (using his share) but $P_{1}$ can prevent other players from learning the secret by not publicly revealing his share.

Let $t, n$ be as above, and let $t^{*} \geq t$ denote the number of players present when the secret is to be reconstructed. Given the above discussion, we can thus conclude the following about the game-theoretic equilibria of "standard" Shamir secret sharing in the above situation (definitions of Nash equilibria and weakly dominating strategies are given in Section 2):

- For any $t, n, t^{*}$, it is a Nash equilibrium for no one to reveal their share.

- If $t^{*}>t$, it is a Nash equilibrium for all $t^{*}$ participating players to reveal their shares. However, as discussed above, it is a weakly dominating strategy for each player not to reveal his share; thus, the Nash equilibrium likely to be reached is the one mentioned earlier in which no one reveals their share.

- If $t^{*}=t$, then having all $t^{*}$ participating players reveal their shares is not even a Nash equilibrium, since each player can profitably deviate by not revealing his share.

Thus, Shamir's protocol with the trivial reconstruction procedure does not suffice in the presence of rational players. Does there exist any protocol for reconstructing the secret in which it is in rational players' best interests to follow the protocol? Generalizing the argument above, Halpern and Teague rule out any protocol terminating in a fixed number of rounds. (Essentially, the above argument is applied to the last round and then backwards induction is used.) This leaves open the possibility of probabilistic protocols without a fixed upper bound on their round complexity, and indeed Halpern and Teague show the existence of such protocols for $t, n \geq 3$. In contrast, they claim a solution is impossible for $n=2$ even if probabilistic protocols are allowed.

\subsection{Our Results}

We revisit the question of rational secret sharing, in the model of Halpern and Teague [7]. As perhaps our most surprising result, we show a simple, probabilistic protocol for $n=2$ parties to reconstruct a shared secret, thus disproving the claim of Halpern and Teague mentioned earlier. Interestingly, the proof given by Halpern and Teague appears to be correct; the problem is that their assumptions regarding the types of protocols that might be used are too restrictive (and are not implied by the model). By relaxing their assumptions in a manner consistent with the model of rational secret sharing they introduce, we are able to circumvent their impossibility result.

Our protocol generalizes in a straightforward way to the case of $n \geq 3$ and arbitrary $t$. Although Halpern and Teague also claim a general solution of this sort, our solution is much simpler. Furthermore, for $n>3$ our solution has a number of advantages as compared with the solution offered by Halpern and Teague; perhaps most importantly, our solution eliminates a second (undesirable) equilibrium that is present in the Halpern-Teague protocol. Other advantages of our approach are summarized in Section 3.3.

Both the Halpern-Teague protocol and our protocol (as initially described) require the continual, periodic involvement of the dealer. At best, this is inconvenient; at worst, this calls into question the motivation for the problem in the first place. We show in Section 4 an intuitively simple way 
to avoid the involvement of the dealer (after the initial share distribution phase) that applies in all scenarios considered here.

As in [7], our techniques extend to the more general case of rational players trying to securely compute an arbitrary function of their inputs, under certain assumptions on the utilities of the players. See Section 5 for further details.

\subsection{Related Work}

There has been much interest of late in bridging cryptography (in which guarantees are provided in the face of worst-case adversarial behavior) and game theory (which concerns itself only with rational deviations). A point to bear in mind is that neither the cryptographic or the gametheoretic model is strictly stronger than the other: typical cryptographic protocols tolerate arbitrary malicious behavior under the assumption that some fraction of the players will follow the protocol exactly as specified; game-theoretic protocols are designed to tolerate "only" rational behavior but do not assume any completely honest players.

Besides the work of Halpern and Teague, the most relevant prior work is the recent sequence of papers by Lepinski, et al. [9, 10] and Izmalkov, et al. [8]. Lepinski, Micali, Peikert, and Shelat [9] show a protocol for completely fair secure function evaluation (SFE), in which all players receive output if any player receives output, even if up to $n-1$ players are malicious. In "standard" communication networks this is known to be impossible [3], and therefore Lepinski, et al. rely on the physical assumption of "secure envelopes" (see the discussion in [9] for the exact properties these should satisfy) to achieve their result. They then show how to use any protocol for completely fair SFE to implement cheap talk in the presence of malicious coalitions; basically, this enables players to reach a correlated equilibrium without having to rely on any external trusted party.

The work of Lepinski, Micali, and Shelat [10] and Izmalkov, Micali, and Lepinski [8] deals (directly or indirectly) with mechanisms for preventing coalitions in the first place. More specifically, these works are concerned with eliminating covert (e.g., steganographic) channels in the secure computation protocol itself so as to prevent signaling between players. Again, they achieve this by relying on physical assumptions (secure envelopes and, in the case of [8], ballot boxes) in addition to standard communication channels. A consequence of the work of Izmalkov, et al. (indeed, the main motivation for their work) is a protocol $\Pi$ for securely implementing any mediated game $\Gamma$ such that (informally) any equilibrium in $\Gamma$ corresponds to an equilibrium in $\Pi$, and vice versa.

Comparison to our work. The work of Lepinski, et al. [9] as well as that of Izmalkov, et al. [8] both offer different solutions to the problems we consider here. Specifically:

- Completely fair SFE [9] guarantees (roughly speaking) that all players learn the output if any player learns the output. This clearly implies a solution for rational secret sharing (even in the presence of collusion), and can also be used to solve the problem of rational $\mathrm{SFE}^{2}$ under certain assumptions on player utilities.

- Since rational secret sharing can be implemented as a mediated game, the work of [8] gives a solution to the problem (without any mediator). Their work is in fact much more general, as it implies a protocol for rational SFE for arbitrary player utilities and even in the presence of coalitions.

The main difference in our work is that we give intuitively-simple and/or very efficient protocols at the expense of providing weaker guarantees. Specifically, we focus only on single-player deviations

\footnotetext{
${ }^{2}$ There are numerous definitions of rational SFE, and so everything we say in this section is somewhat informal.
} 
(and do not handle collusion), and also make specific assumptions regarding the utilities of the players. Under these assumptions, our protocol for general secure function evaluation in Section 5 can be viewed as either a weak form of rational SFE, or completely fair SFE in the presence of rational (rather than arbitrarily malicious) parties.

An additional important difference between our work and that of $[9,10,8]$ is that we rely on weaker assumptions with respect to the model of communication. Instead of relying on "secure envelopes" and "ballot boxes" as in $[9,10,8]$ — which seem to be difficult primitives to realize unless parties are physically co-located - our solutions rely on standard communication channels with the exception that, as in [7], we assume simultaneous broadcast whereby each party broadcasts a message at the same time. (Equivalently, we do not allow "rushing.") Whether one finds the assumption of simultaneous broadcast realistic or not, we note that it is a strictly weaker assumption than secure envelopes or ballot boxes since simultaneous broadcast can be constructed from either of the latter but not vice versa.

Concurrent work. Concurrently and independently of our work, Abraham, et al. [1] and Lysyanskaya and Triandopoulos [11] consider problems related to those considered here. Abraham, et al. also note the flaw in the Halpern-Teague impossibility claim for $n=2$, and show a solution for this case which is similar in spirit to our own. Their primary contribution is to define a notion of resistance to coalitions of rational players and to show a coalition-resistant protocol; we remark that our protocols are resistant to coalitions in the same sense as theirs. Lysyanskaya and Triandopoulos examine the case of "mixed" security when both arbitrarily malicious and rational players might be present. (It seems that the work of Abraham, et al. [1] can be extended to this case by modeling arbitrarily-malicious players as players whose utility is unknown. Proving this rigorously remains a subject for future research.) Both papers also show, under certain conditions, how protocols can be designed without exact knowledge of players' utilities. Interestingly (and somewhat serendipitously!), both these works, as well as our own, rely on essentially the same underlying techniques.

\section{Definitions for Rational Secret Sharing}

We briefly review the model of rational secret sharing we assume in this paper. Our model is intended to match the model used by Halpern and Teague, though there are many details they do not make explicit.

As discussed earlier, we have a dealer $D$ holding a secret $s$, and $n$ players $P_{1}, \ldots, P_{n}$. There is also a threshold $t \leq n$, known to all players, which is fixed at the outset. A protocol proceeds in a sequence of iterations, where each iteration may consist of multiple communication rounds. At the beginning of each iteration, $D$ distributes some information (privately) to each of the $n$ players; at this point, no subset of fewer than $t$ players should have any information about $s$. During an iteration, the dealer does not take part in the protocol. Instead, some set of $t^{*} \geq t$ "active" players, all of whom are assumed to be rational, run the protocol amongst themselves by simultaneously broadcasting messages in a series of rounds. (Halpern and Teague additionally allow private communication between the players but we do not need this.) We assume the same set of $t^{*}$ players runs the protocol in every iteration (this assumption is not strictly necessary, but without this assumption we would need to specify how a player learns what happened in an iteration in which he was not involved). We also assume that each of these $t^{*}$ players knows the set of active players. At the end of an iteration, the protocol either terminates or proceeds to the next iteration. We assume the dealer is honest, and follows the protocol as specified. To rule out trivial protocols, 
we require that if $t^{*} \geq t$ players follow the protocol in each iteration, then the secret is eventually reconstructed (with probability 1 ).

We stress that broadcast in a given round is assumed to occur simultaneously for all players; that is, we do not allow "rushing" as in the standard literature on secure multi-party computation. Rational secret sharing is easily seen to be impossible if rushing is allowed: all players will simply wait to see what other players do, and no one will ever broadcast anything.

In the above description, as in [7], the dealer is assumed to be involved at the beginning of each iteration. In Section 4, we show that it is possible for the dealer to be involved only once at the beginning of the protocol.

We let $\sigma_{i}$ denote the (possibly randomized) strategy employed by player $P_{i}$, and let $\vec{\sigma}=$ $\left(\sigma_{1}, \ldots, \sigma_{n}\right)$ denote the vector of players' strategies. Following standard game-theoretic notation, we let $\left(\sigma_{i}^{\prime}, \vec{\sigma}_{-i}\right) \stackrel{\text { def }}{=}\left(\sigma_{1}, \ldots, \sigma_{i-1}, \sigma_{i}^{\prime}, \sigma_{i+1}, \ldots, \sigma_{n}\right)$; that is, $\left(\sigma_{i}^{\prime}, \vec{\sigma}_{-i}\right)$ denotes the strategy vector $\vec{\sigma}$ with $P_{i}$ 's strategy changed to $\sigma_{i}^{\prime}$.

Let $\mu_{i}(o)$ denote the utility of player $P_{i}$ for the outcome $o$. For a particular outcome $o$ of the protocol, we let $\delta_{i}(o)$ be a bit denoting whether or not $P_{i}$ learns the secret, and let num $(o)=$ $\sum_{i} \delta_{i}(o)$; i.e., num $(o)$ is simply the number of players who learn the secret. Following [7], we make the following assumptions about the utility functions of the players:

- $\delta_{i}(o)>\delta_{i}\left(o^{\prime}\right) \Rightarrow \mu_{i}(o)>\mu_{i}\left(o^{\prime}\right)$.

- If $\delta_{i}(o)=\delta_{i}\left(o^{\prime}\right)$, then $\operatorname{num}(o)<\operatorname{num}\left(o^{\prime}\right) \Rightarrow \mu_{i}(o)>\mu_{i}\left(o^{\prime}\right)$.

That is, player $P_{i}$ first prefers outcomes in which he learns the secret; as long as $\delta_{i}$ remains constant, player $P_{i}$ prefers strategies in which the fewest number of other players learn the secret. We let $U_{i}(\vec{\sigma})$ denote the expected value of the utility of $P_{i}$ under strategy vector $\vec{\sigma}$, and assume that rational players wish to maximize this value.

Our notion of a protocol corresponds to a game along with a prescribed strategy vector $\vec{\sigma}$. As in [7], we are interested in protocols whose prescribed strategy vector $\vec{\sigma}$ corresponds to a Nash equilibrium that survives iterated deletion of weakly dominated strategies. We review these definitions briefly, and refer the reader to [12, 7] for more extensive discussion.

Definition $1 \mathrm{~A}$ vector of strategies $\vec{\sigma}$ is a Nash equilibrium if the following holds for all $i$ : for any $\sigma_{i}^{\prime} \neq \sigma_{i}$, we have $U_{i}\left(\sigma_{i}^{\prime}, \vec{\sigma}_{-i}\right) \leq U_{i}(\vec{\sigma})$.

That is, given that all other players are following $\vec{\sigma}_{-i}$, there is no incentive for $P_{i}$ to deviate and follow any strategy other than $\sigma_{i}$.

In general, multiple Nash equilibria may exist. An inherently "unstable" Nash equilibrium (i.e., one unlikely to be reached) is one in which any of the players' strategies are weakly dominated by other strategies. Informally, a strategy $\sigma_{i}$ of player $P_{i}$ is weakly dominated by another strategy $\sigma_{i}^{\prime}$ if (1) $P_{i}$ is sometimes better off playing $\sigma_{i}^{\prime}$ than playing $\sigma_{i}$, and (2) $P_{i}$ is never worse off playing $\sigma_{i}^{\prime}$ than playing $\sigma_{i}$. Recalling the example from the introduction, say a secret is shared using $t$-outof- $n$ secret sharing (with $t<n$ ) and consider the strategy vector in which all $n$ players reveal their shares. This is a Nash equilibrium: the secret is reconstructed even if any single player deviates. On the other hand, for each player $P_{i}$, revealing the share is weakly dominated by not revealing the share: if fewer than $t-1$ other players or more than $t-1$ other players reveal their shares, then nothing changes; if exactly $t-1$ other player reveal their shares then $P_{i}$ learns the secret but no one else does. Formal definitions follow.

Definition 2 Let $S_{i}$ denote a set of strategies for $P_{i}$, and let $S_{-i} \stackrel{\text { def }}{=} S_{1} \times \cdots \times S_{i-1} \times S_{i+1} \cdots \times S_{n}$. A strategy $\sigma_{i} \in S_{i}$ is weakly dominated by a strategy $\sigma_{i}^{\prime} \in S_{i}$ with respect to $S_{-i}$ if (1) there 
exists a $\vec{\sigma}_{-i} \in S_{-i}$ such that $U_{i}\left(\sigma_{i}, \vec{\sigma}_{-i}\right)<U_{i}\left(\sigma_{i}^{\prime}, \vec{\sigma}_{-i}\right)$ and (2) for all $\vec{\sigma}_{-i} \in S_{-i}$, it holds that $U_{i}\left(\sigma_{i}, \vec{\sigma}_{-i}\right) \leq U_{i}\left(\sigma_{i}^{\prime}, \vec{\sigma}_{-i}\right)$.

Strategy $\sigma_{i}$ is weakly dominated with respect to $S_{-i}$ if there exists a $\sigma_{i}^{\prime} \in S_{i}$ such that $\sigma_{i}$ is weakly dominated by $\sigma_{i}^{\prime}$ with respect to $S_{-i}$.

Definition 3 Let $\operatorname{DOM}_{i}\left(S_{1} \times \cdots \times S_{n}\right)$ denote the set of strategies in $S_{i}$ that are weakly dominated with respect to $S_{-i}$. Let $S_{i}^{0}$ denote the initial set of allowable strategies of $P_{i}$. For all $k \geq 1$, define $S_{i}^{k}$ inductively as $S_{i}^{k} \stackrel{\text { def }}{=} S_{i}^{k-1} \backslash \operatorname{DOM}_{i}\left(S_{1}^{k-1} \times \cdots \times S_{n}^{k-1}\right)$. Let $S_{i}^{\infty} \stackrel{\text { def }}{=} \cap_{k} S_{i}^{k}$.

We say $\sigma_{i}$ survives iterated deletion of weakly dominated strategies if $\sigma_{i} \in S_{i}^{\infty}$.

\section{Protocols for Rational Secret Sharing}

We review the Halpern-Teague solution, and then describe our protocol. We conclude with some discussion of the relative merits of our approach.

\subsection{The Halpern-Teague Solution}

We provide a high-level overview of the solution of Halpern and Teague for 3-out-of-3 secret sharing. We later discuss how they propose to generalize their solution for $n>3$ and $t \geq 3$.

The Halpern-Teague protocol in the 3-out-of-3 case proceeds as follows: at the beginning of each iteration, the dealer runs a fresh invocation of the Shamir secret-sharing scheme and sends the appropriate shares to each player. (Actually, a simpler additive secret-sharing scheme could also be used.) During an iteration, each player $P_{i}$ flips a biased coin $c_{i}$ which is equal to 1 with some probability $\alpha$. The players then run what is essentially an information-theoretically secure multiparty computation protocol to compute the value $c^{*}=\bigoplus c_{i}$. (Here is where Halpern and Teague need to assume the existence of private channels between the players.) In particular, it is impossible for any player to cheat (except for aborting the protocol; see below), or to learn information about the $\left\{c_{i}\right\}$ values of the other parties that is not implied by $c^{*}$. If $c^{*}=c_{i}=1$, player $P_{i}$ broadcasts his share. If all shares are revealed, the secret is reconstructed and the protocol ends. If $c^{*}=1$ and either no shares or exactly two shares are revealed, or if the secure computation of $c^{*}$ was aborted, then all players refuse to run the protocol from then on (and so, effectively, the protocol is terminated). In any other case, players proceed to the next iteration.

Note that the secret is only reconstructed if $c_{1}=c_{2}=c_{3}=1$. Thus, assuming players act honestly, the expected number of iterations until the protocol terminates is $\alpha^{-3}$.

To see intuitively why the above gives a Nash equilibrium, assume $P_{1}, P_{2}$ follow the protocol and consider whether $P_{3}$ should deviate. First note that there is no incentive for $P_{3}$ to bias $c_{3}$ to be 0 with higher probability, since when $c_{3}=0$ at least one of $P_{1}, P_{2}$ will not broadcast their shares in that iteration. There is also no incentive for $P_{3}$ to bias $c_{3}$ to be 1 with higher probability, either: although this may cause the secret to be reconstructed sooner, it will have no effect on $P_{3}$ 's utility. It is also easy to see that, given $c^{*}=0$ or $c_{3}=0$, there is no incentive for $P_{3}$ to deviate from the protocol. Finally, when $c^{*}=c_{3}=1$, player $P_{3}$ does not know whether $c_{1}=c_{2}=1$ (which occurs with probability $\frac{\alpha^{2}}{\alpha^{2}+(1-\alpha)^{2}}$ ) or $c_{1}=c_{2}=0$ (which occurs with the remaining probability). Thus, if $P_{3}$ does not broadcast its share it runs the risk of having the protocol terminate without ever learning the secret. If $\alpha$ is set appropriately based on $P_{3}$ 's utility function, it can be shown that it is not in $P_{3}$ 's best interest to deviate.

For $n>3$ and $t \geq 3$, Halpern and Teague suggest the following: of the $t^{*} \geq t$ players who are present, $t$ players are designated. Players are split into 3 groups, such that there is at least 
one designated player in each group. One designated player in each group is chosen as a leader. The designated players send their shares to the leader of their group, and then the leaders run essentially the 3-out-of-3 solution described above. (When the leaders are supposed to broadcast, they broadcast the shares of all the players in their group in such a way that all $t^{*}$ players can hear.)

Halpern and Teague also describe a solution for 2-out-of- $n$ secret sharing for $n \geq 3$, but in this case they require that the number of participating players $t^{*}$ is strictly greater than 2 (and so this solution does not satisfy the model as we have described it here).

\subsection{Our Solution}

Recall that Halpern and Teague claim that rational secret sharing is impossible when $n=2$. In their impossibility proof, however, they implicitly assume that the dealer is limited to sending valid shares of the secret to the players at the beginning of each iteration. They therefore focus only on possible actions of the players during an iteration. We see no reason to impose any such restriction on the dealer's actions; note that the model, as described earlier, does not impose any such restriction. As we show in this section, once this assumption is removed a solution is possible even when $n=2$, and things become simpler in the case of general $t, n$.

Specifically, consider the following protocol for the case of arbitrary $t, n$ : say the dealer holds a secret $s$ which lies in a strict subset $S$ of a finite field $\mathbb{F}$ (if $s$ lies in some field $\mathbb{F}^{\prime}$, this is easy to achieve by taking a larger field $\mathbb{F}$ containing $\mathbb{F}^{\prime}$ as a subfield). We assume players know $S$. At the beginning of each iteration, with probability $\beta$ the dealer generates a random $t$-out-of- $n$ Shamir sharing of $s$, and with probability $1-\beta$ the dealer generates a random $t$-out-of- $n$ Shamir sharing of an arbitrary element $\hat{s} \in \mathbb{F} \backslash S$; we describe how $\beta$ is chosen below. These shares are distributed to the players. Note that no player can tell from their share whether the players were given a share of $\hat{s}$ or the true secret $s$.

Each player begins with a flag all_honest $=$ true. During an iteration, each of the $t^{*}$ active players then does the following: First, if all_honest $=$ true then broadcast the share received from the dealer. (Otherwise, do nothing.) Then:

- If at least $t$ shares were broadcast, a value $s^{\prime}$ is reconstructed, and $s^{\prime} \in S$ then $s^{\prime}$ is the secret and so this player can terminate their execution of the protocol. (The player should then inform the dealer that the secret was reconstructed so the dealer can terminate the protocol.)

- If $t^{*}$ shares were broadcast (i.e., all players broadcast their shares), a value $s^{\prime}$ is reconstructed, and $s^{\prime} \in \mathbb{F} \backslash S$, then this is an invalid secret. In this case simply proceed to the next iteration.

- In any other case (i.e., at least one player failed to broadcast their share, and the real secret could not be constructed from the remaining shares), set all honest $:=$ false and proceed to the next iteration.

Note that once a player fails to broadcast their share in one iteration, all other players are supposed to simply do nothing for the remainder of the protocol. ${ }^{3}$ We stress that the protocol does not automatically end once a player refuses to broadcast their share, since we do not wish to assume that the dealer monitors what happens during an iteration. Another option would be for

\footnotetext{
${ }^{3}$ Doing nothing for the remainder of the protocol is essentially equivalent to terminating. The only differences are that (1) a player can still listen to messages broadcast by players (in the hopes that the secret will be reconstructed); and (2) the dealer will continue running the protocol forever. This latter is a bit unsatisfying, but is necessary for the analysis.
} 
players to inform the dealer once some player does not broadcast their share, but in fact it is easy to see that such action is weakly dominated by not informing the dealer.

Theorem 1 For appropriate choice of $\beta$, the above protocol constitutes a Nash equilibrium for $t$-out-of-n secret sharing that survives iterated deletion of weakly dominated strategies.

Proof We first consider the case of $t=n=2$, and then discuss how to generalize the proof for arbitrary $t, n$. It is not hard to see that the protocol is a Nash equilibrium for appropriate choice of $\beta$ : Say $P_{2}$ acts according to the protocol and consider whether $P_{1}$ has any incentive to deviate. Without loss of generality, consider a deviation in the first iteration. The only possible deviation is for $P_{1}$ to refuse to broadcast his share. In this case, he learns the secret (while $P_{2}$ does not) with probability $\beta$, but with probability $1-\beta$ he will never learn the secret.

Say $P_{1}$ 's utility is $U^{+}$if he learns the secret but $P_{2}$ does not; $U$ if both players learn the secret; and $U^{-}$if neither player learns the secret, where $U^{+}>U>U^{-}$. If $P_{1}$ follows the protocol, his expected utility is $U$. If $P_{1}$ deviates, his expected utility is $\beta \cdot U^{+}+(1-\beta) \cdot U^{-}$. So as long as

$$
U>\beta \cdot U^{+}+(1-\beta) \cdot U^{-},
$$

it is in $P_{1}$ 's best interest to follow the protocol. For appropriate $\beta \in(0,1)$, then, the strategy profile in which both parties follow the protocol is a Nash equilibrium.

It is immediate that the same analysis holds for general $t, n$, regardless of the number of participating players $t^{*}$.

We next prove that our protocol survives iterated deletion of weakly dominated strategies. It is easy to see that once a player learns the real secret, not terminating the protocol is weakly dominated by terminating the protocol (i.e., never broadcasting another message); furthermore, when a player does not learn the real secret, telling the dealer (falsely) that the secret was reconstructed is weakly dominated by not doing so. The first round of iterated deletion thus leaves only strategies in which (1) players always terminate the protocol once they learn the secret, and (2) players never falsely tell the dealer that they learned the secret (thereby terminating the protocol) when in fact they have not.

We show that no other deterministic strategies are weakly dominated (and hence no randomized strategies are weakly dominated either). We again begin with the case $t=n=2$. We show that for all deterministic strategies $\sigma, \sigma^{\prime}$ of $P_{1}$, there exist (not-yet-deleted) strategies $\tau, \tau^{\prime}$ of $P_{2}$ such that $U_{1}(\sigma, \tau)>U_{1}\left(\sigma^{\prime}, \tau\right)$ but $U_{1}\left(\sigma, \tau^{\prime}\right)<U_{1}\left(\sigma^{\prime}, \tau^{\prime}\right)$. This proves that all remaining deterministic strategies of $P_{1}$ are incomparable, and so none are ever deleted.

Let $h_{i}(\sigma, \tau ; r)$ denote the view of $P_{1}$ up to the point when $P_{1}$ has to make its move in iteration $i$, given the indicated strategies $\sigma$ and $\tau$ and assuming the dealer uses coins $r$ (we view $r$ as an infinite string encoding the dealer's random choices in all iterations). That is, $h_{i}(\sigma, \tau ; r)$ includes the actions of $P_{2}$ in iterations 1 through $i-1$ as well as the information sent by the dealer to $P_{1}$ in iterations 1 through $i$. Let $A_{i}(\sigma, \tau ; r)$ denote the action taken by $P_{1}$ in iteration $i$, again for the indicated strategies and random coins. We say a player cooperates in some iteration if they reveal their share, and defects if they do not.

Now take arbitrary deterministic strategies $\sigma \neq \sigma^{\prime}$ for $P_{1}$ that have not yet been deleted. Let $\tau^{0}$ be a strategy of $P_{2}, i \geq 1$ be an integer, and $r$ a set of coins such that

$$
h_{i-1}\left(\sigma, \tau^{0} ; r\right)=h_{i-1}\left(\sigma^{\prime}, \tau^{0} ; r\right)
$$

but

$$
A_{i}\left(\sigma, \tau^{0} ; r\right) \neq A_{i}\left(\sigma^{\prime}, \tau^{0} ; r\right)
$$


i.e., iteration $i$ is the first iteration in which the actions of $P_{1}$ differ. Note that some such $\tau^{0}, i, r$ must exist or else $\sigma=\sigma^{\prime}$. Note also that $P_{1}$ could not have reconstructed the real secret in any prior iteration, since otherwise $P_{1}$ would defect (no matter what) in iteration $i$. Without loss of generality, assume $A_{i}\left(\sigma, \tau^{0} ; r\right)$ is to defect and $A_{i}\left(\sigma^{\prime}, \tau^{0} ; r\right)$ is to cooperate.

The actions of $P_{1}$ in iteration $i$ depend only on $h_{i}\left(\sigma, \tau^{0} ; r\right)$. In particular, the actions of $P_{1}$ in iteration $i$ cannot depend on whether or not the dealer shared the real secret or the fake secret in that iteration. Furthermore, in any prior iteration in which $P_{1}$ cooperated and $P_{2}$ defected, $P_{1}$ cannot determine from $h_{i}\left(\sigma, \tau^{0} ; r\right)$ whether or not $P_{2}$ was able to reconstruct the real secret in that iteration. Thus, given $r$ for which Equations (1) and (2) hold, we may find another $r$ for which these equations still hold but in which $P_{2}$ has not reconstructed the real secret by iteration $i$. We can therefore simply assume that $r$ already satisfies this condition.

Consider the following strategy $\tau$ of $P_{2}$ : (1) act identically to $\tau^{0}$ through iteration $i-1 ;(2)$ in iteration $i$, defect; (3) in all subsequent iterations: if $P_{1}$ defected in iteration $i$, then cooperate; if $P_{1}$ cooperated in iteration $i$, defect. For any $r$ as discussed above, $P_{1}$ is clearly better off playing $\sigma$ than $\sigma^{\prime}$ against strategy $\tau$.

Next consider the following strategy $\tau^{\prime}$ of $P_{2}$ : (1) act identically to $\tau^{0}$ through iteration $i-1$; (2) in iteration $i$, cooperate; (3) in all subsequent iterations (assuming $P_{2}$ has not yet reconstructed the real secret): if $P_{1}$ defected in iteration $i$, then defect; if $P_{1}$ cooperated in iteration $i$, cooperate. Exactly as when we argued earlier that our protocol was a Nash equilibrium, we have $U_{1}\left(\sigma, \tau^{\prime}\right)<$ $U_{1}\left(\sigma^{\prime}, \tau^{\prime}\right)$. (Here, $P_{1}$ is not better off playing $\sigma^{\prime}$ than $\sigma$ for all $r$ as discussed above; however, $P_{1}$ is better off playing $\sigma^{\prime}$ in expectation.)

The same argument extends to the case of general $t, n$, regardless of the number of participating players $t^{*}$. We simply replace $\tau^{0}$ with a strategy profile of $n-1$ strategies such that Equations (1) and (2) above are still valid, and then define $\tau$ and $\tau^{\prime}$ as above, but modifying the strategies of all other players.

We remark that when $t^{*}=t$ our protocol has no additional Nash equilibrium which is preferred, by any player, to the prescribed equilibrium.

\subsection{Discussion}

Our approach has a number of advantages as compared to [7]:

- Most obvious, we circumvent their impossibility result for the case $n=2$. We also show an admissible solution for the 2-out-of- $n$ case.

- Our protocol is (in our opinion) much simpler than the Halpern-Teague protocol. This is true for all settings of $t, n$, but is especially true for the case of $n>3, t \geq 3$ where the Halpern-Teague protocol requires players to somehow delegate specific roles and select group leaders.

- Our protocol requires only a broadcast channel, in contrast to the Halpern-Teague protocol which relies on private channels in addition to broadcast.

- At least for the case $t^{*}=t$ (which is always the case when $t=n$ ), our protocol has no "undesirable" Nash equilibria. This is in contrast to the Halpern-Teague solution for general $n$, where there is the undesirable equilibrium in which the three "group leaders" pool the shares they receive from all the designated players and reconstruct the secret only amongst themselves. 


\section{Removing the Dealer}

A drawback of both our protocol (as described in the previous section) as well as that of Halpern and Teague is that the dealer must be involved at the beginning of every iteration. It would be much nicer to have a solution that works exactly like standard secret sharing, where the dealer is involved only once at the beginning of the protocol.

We sketch here a conceptually simple (though inefficient) way to avoid continual involvement of the dealer while still ensuring that parties eventually reconstruct the secret with probability 1 . Our idea applies both to our protocol and that of Halpern and Teague, but for simplicity we describe it in the context of our protocol only. The protocol proceeds as follows:

Setup: To share a secret $s$, the dealer prepares a valid $t$-out-of- $n$ Shamir sharing $\left\{s_{i}\right\}$ of $s$. The dealer also generates a signature $\sigma_{i}$ on each share $s_{i}$ with respect to a publicly-known verification key $P K$ (alternately, $P K$ can simply be sent to each player). The dealer sends $\left(s_{i}, \sigma_{i}\right)$ to player $P_{i}$. Each player begins with all _honest $=$ true.

The protocol: At the beginning of each iteration, the players proceed as follows:

1. The $t^{*}$ participating parties run a secure computation protocol $[15,6,5]$ secure against one malicious player. The protocol computes the following probabilistic functionality:

- Each party inputs the values $\left(s_{i}, \sigma_{i}\right)$ received from the dealer. The functionality checks that each $\sigma_{i}$ is a valid signature on $s_{i}$ (with respect to the dealer's public key $P K$ ), and aborts if this is not the case.

- The $t^{*} \geq t$ input shares define a secret $s$. With probability $\beta$, the functionality generates a fresh $t$-out-of- $n$ Shamir sharing $\left\{s_{i}^{\prime}\right\}$ of $s$, and each player $P_{i}$ receives output $s_{i}^{\prime}$.

- With probability $1-\beta$, the functionality generates a fresh $t$-out-of- $n$ Shamir sharing $\left\{s_{i}^{\prime}\right\}$ of a bogus secret $\hat{s} \in \mathbb{F} \backslash S$, and each player $P_{i}$ receives output $s_{i}^{\prime}$.

This protocol is run regardless of the value of all_honest.

2. If cheating is detected in the secure computation protocol above (i.e., the secure computation protocol is aborted), set all_honest $:=$ false.

3. Next, parties proceed as in the previous section; specifically, if all honest $=$ true then a player broadcasts the output $s_{i}^{\prime}$ he received from the secure computation protocol. ${ }^{4}$ If this enables reconstruction of a secret $s^{\prime} \in S$, the protocol terminates and the true secret has been reconstructed. If some player refused to broadcast their output share, then each party sets all_honest $:=$ false. In any other case, players erase the $\left\{s_{i}^{\prime}\right\}$ and proceed to the next iteration (using $\left(s_{i}, \sigma_{i}\right)$ as before).

A subtlety (which applies also in the following section) is the question of whether security of the secure computation protocol used above should hold information-theoretically or whether computational security suffices. In the former case, an argument similar to that used in the previous section shows that - under appropriate conditions on $\beta$ - the above protocol is a Nash equilibrium

\footnotetext{
${ }^{4}$ Actually, to prevent players from broadcasting a modified value for $s_{i}^{\prime}$, it is necessary to have the functionality authenticate the $\left\{s_{i}^{\prime}\right\}$ in some way. There are many ways to do this. Perhaps the conceptually-simplest solution is to have the dealer also distribute shares of his secret signing key in a $t$-out-of- $n$ manner among the players. Then the functionality can also generate valid dealer signatures on the $\left\{s_{i}^{\prime}\right\}$ (the iteration number should also be signed to prevent replay of an earlier output value). We omit any further details for simplicity.
} 
surviving iterated domination of weakly dominated strategies. To implement such a solution, however, we need the additional assumption of private channels between the players.

If a computationally-secure protocol is used, one way to proceed is to work in a concrete setting: that is, assume all players are limited to running for at most $t$ steps (in some fixed computational model); assume the protocol is secure (defined appropriately) except with some (small) probability $\epsilon$ against adversaries running in time $t$; and then modify the analysis appropriately. Rigorously formalizing this is left for future work. See [11] for a slightly different approach.

\section{General Secure Function Evaluation}

The techniques outlined above generalize to the case of the secure computation of an arbitrary function $f$. In this sense, they yield a protocol for a weak notion of completely fair SFE [9] requiring that (1) all players are rational; and (2) players' utility functions are such that they all prefer to learn the output. (In contrast, the work of [9] shows a protocol for completely fair SFE tolerating malicious players, but under a stronger assumption on the available communication. See Section 1.2.) We also assume (as in $[7,1,11]$ ) that players prefer that their own inputs remain private (other than what is leaked by evaluation of $f$ ).

To compute the (possibly randomized) single-output function $f$ :

1. Let $f^{\prime}$ be the following (multi-output, randomized) function: on inputs $x_{1}, \ldots, x_{n}$, compute $y \leftarrow f\left(x_{1}, \ldots, x_{n}\right)$. Then generate a random $t$-out-of- $n$ Shamir sharing $\left(s_{1}, \ldots, s_{n}\right)$ of the result $y$, and give output $s_{i}$ to player $P_{i}$.

2. Players run a secure computation protocol for $f^{\prime}$, and obtain outputs $s_{1}, \ldots, s_{n}$. If this protocol is aborted, all players terminate the entire protocol and the output is never reconstructed.

3. As in the previous section, players compute a functionality that takes as input ${ }^{5}\left(s_{1}, \ldots, s_{n}\right)$ and, with probability $\beta$ computes a random Shamir sharing $\left\{s_{i}^{\prime}\right\}$ of the value $y$ these shares define, and with probability $1-\beta$ computes a random Shamir sharing $\left\{s_{i}^{\prime}\right\}$ of some default value not in the range of $f$. Each player $P_{i}$ receives output $s_{i}^{\prime}$. If this protocol is aborted, all players terminate the entire protocol and the output is never reconstructed.

4. Players simultaneously broadcast the $s_{i}^{\prime}$ and reconstruct the value $s^{\prime}$ these shares define. If some player did not broadcast a (valid) share, then all players terminate the protocol and do not participate in any future iterations. If $s^{\prime}$ is in the range of $f$ then $y=s^{\prime}$ is the desired output and the protocol is done; in any other case, players proceed to the next iteration.

The protocol can be suitably generalized for the case where $f$ outputs a vector of values, one for each player.

We remark that, as in standard formulations of secure multi-party computation, players who choose not to follow the protocol may change their "true" inputs to an arbitrary other value. (I.e., a player $P_{i}$ with "true" input $x_{i}$ may cause $f\left(x_{1}, \ldots, x_{i}^{\prime}, \ldots, x_{n}\right)$ to be evaluated for arbitrary $x_{i}^{\prime}$.) For rational players, this may occur if a player would prefer to change his input value even if a completely incorruptible third party were to evaluate $f$ based on inputs given to it by the players. Shoham and Tennenholtz [14] define the class of NCC functions and argue that if $f$ is an NCC function then no rational player has any incentive to modify their inputs. It seems to us, however,

\footnotetext{
${ }^{5}$ As before, there is the issue of authenticating the shares $s_{1}, \ldots, s_{n}$ provided as input to this functionality. This can be handled in a similar manner as before.
} 
that there are some subtle problems with the way NCC functions are defined there. We leave further exploration of this issue for future study.

\section{Conclusions}

We have provided a new approach to rational secret sharing and secure computation that improves, in many respects, on an earlier solution of Halpern and Teague. Our work also offers an alternate approach to the generic (and more powerful) solutions of $[9,8]$ : our protocols are simpler, and rely on weaker assumptions regarding the communication between players.

\section{Acknowledgments}

We thank Silvio Micali for clarifications regarding the relationship between our work and $[9,10,8]$, as well as for prompting us to think about using our techniques to obtain rational SFE. We would also like to thank Joe Halpern for his helpful comments regarding our proof of Theorem 1.

\section{References}

[1] I. Abraham, D. Dolev, R. Gonen, and J. Halpern. Distributed Computing Meets Game Theory: Robust Mechanisms for Rational Secret Sharing and Multiparty Computation. 25th ACM Symposium on Principles of Distributed Computing (PODC 2006), to appear.

[2] G.R. Blakley. Safeguarding Cryptographic Keys. National Computer Conference, vol. 48, pp. 313-317, AFIPS Press, 1979.

[3] R. Cleve. Limits on the Security of Coin Flips when Half the Processors are Faulty. 18th Annual ACM Symposium on Theory of Computing (STOC 1986).

[4] D. Fudenberg and J. Tirole. Game Theory. MIT Press, 1991.

[5] O. Goldreich. Foundations of Cryptography, vol. 2: Basic Applications, Cambridge University Press, 2004.

[6] O. Goldreich, S. Micali, and A. Wigderson. How to Play any Mental Game or A Completeness Theorem for Protocols with Honest Majority. 19th Annual ACM Symposium on Theory of Computing (STOC 1987).

[7] J. Halpern and V. Teague. Rational Secret Sharing and Multiparty Computation. 36th Annual ACM Symposium on Theory of Computing (STOC 2004).

[8] S. Izmalkov, S. Micali, and M. Lepinski. Rational Secure Function Evaluation and Ideal Mechanism Design. 46th Annual IEEE Symposium on Foundations of Computer Science (FOCS 2005).

[9] M. Lepinski, S. Micali, C, Peikert, and A. Shelat. Completely Fair SFE and Coalition-Safe Cheap Talk. 23rd ACM Symposium on Principles of Distributed Computing (PODC 2004).

[10] M. Lepinski, S. Micali, and A. Shelat. Collusion-Free Protocols. 37th Annual ACM Symposium on Theory of Computing (STOC 2005). 
[11] A. Lysyanskaya and N. Triandopoulos. Rationality and Adversarial Behavior in Multi-Party Computation. Advances in Cryptology - Crypto 2006, to appear.

[12] M.J. Osborne and A. Rubinstein. A Course in Game Theory. MIT Press, 1994.

[13] A. Shamir. How to Share a Secret. Comm. ACM, 22(11): 612-613 (1979).

[14] Y. Shoham and M. Tennenholtz. Non-Cooperative Computing: Boolean Functions with Correctness and Exclusivity. Theoretical Computer Science 343(1-2): 97-113 (2005).

[15] A. C.-C. Yao. How to Generate and Exchange Secrets. 27th Annual IEEE Symposium on Foundations of Computer Science (FOCS 1986). 